\title{
Kontinuierliche Überwachung von Fermentations- und Kultivierungsprozessen mittels Surface Plasmon Resonance (SPR) Spectroscopy
}

Anja Henseleit ${ }^{1}$, Julia Stürmer ${ }^{2}$, Andreea Tomita ${ }^{1}$, Carolin Pohl ${ }^{1}$, Thomas Bley ${ }^{1}$, Elke Boschke ${ }^{1}$ ${ }^{1} T U$ Dresden Institut für Lebensmittel- und Bioverfahrenstechnik, Dresden, anja.henseleit@tu-dresden.de

${ }^{2}$ EAH Jena

\begin{abstract}
:
Eine prozessbegleitende Überwachung von Fermentations- und Kultivierungsprozessen ermöglicht nicht nur eine zeitnahe Reaktion auf Veränderungen von kritischen Parametern (z. B. Medienzusammensetzung und Metaboliten) sondern verbessert darüber hinaus das Verständnis der Prozesse an sich. Besonders geeignet ist in diesem Zusammenhang die Oberflächenplasmonenresonanz (SPR)-Spektroskopie. Sie misst durch molekulare Interaktionen hervorgerufene Veränderungen des Brechungsindexes und bietet so die Möglichkeit, Analyseproben in Echtzeit und ohne vorhergehende zeitaufwendige Aufbereitung, labelfrei zu charakterisieren. Eine der größten Herausforderungen ist dabei die Etablierung geeigneter Strategien zur Immobilisierung des jeweiligen Rezeptors (z.B. Antikörper) an die Sensoroberfläche als Voraussetzung für die nachfolgende, spezifische Bindung der nachzuweisenden Analyten. Vor allem für Messungen in Realproben ist es entscheidend, dass die funktionalisierte Fläche eine ausreichende Immobilisierungsdichte und eine genügend hohe Bindungsaktivität besitzt, um auch geringe Konzentrationen detektieren zu können.

Für die zellbasierte Substanzcharakterisierung an humanen Leberzellen wird als Marker unter anderem humanes Serumalbumin (HSA) verwendet. Es ist das von der Leber am meisten synthetisierte Protein und kennzeichnet somit die spezifische Aktivität von Leberzellen. Deshalb wird es als Funktionalitätsnachweis von in vitro kultivierten Hepatozyten genutzt. Sie werden in einem, von der TU Berlin und dem Fraunhofer IWS entwickelten Multi-Organ-Chip, für die prädiktive Substanztestung kultiviert. Die SPR-Spektroskopie soll als Detektionsmethode für das HSA dienen.
\end{abstract}

Key words: humanes Serumalbumin (HSA), Surface Plasmon Resonance (SPR), Antikörper, Sandwich-Assay

\section{Einleitung}

Biosensoren halten mehr und mehr Einzug in unserem täglichen Leben. So sind sie mittlerweile aus Bereichen wie der klinischen Diagnostik, der Umweltanalytik, der Militärtechnik, der Lebensmittelanalytik und der Prozesskontrolle nicht mehr wegzudenken.

Generell bestehen Biosensoren aus zwei Komponenten: dem Tansducer zur Signalwandlung und dem Rezeptor zur spezifischen Erkennung des gewünschten Targets [1]. Aufgrund von zahlreichen Vorteilen, wie der zerstörungsfreien Arbeitsweise und der schnellen Signalgenerierung, bilden die optischen Methoden die größte Gruppe der Transducer. Sie messen Veränderungen in der Adsorption, Fluoreszenz, Lumineszenz, Lichtstreuung oder des Brechungsindexes.
Die Oberflächenplasmonenresonanz (SPR)Spektroskopie gehört zu den bevorzugt eingesetzten, optischen Methoden, da sie Wechselwirkungen zwischen zwei Reaktionspartnern auf der Grundlage von BrechungsindexÄnderungen in Echtzeit labelfrei messen kann. Die Detektion des Brechungsindex erfolgt dabei innerhalb eines evaneszenten Feldes, welches sich an der Grenzfläche zwischen einem Metall (typischerweise Gold) und einem Dielektrikum ausbildet. Für die Messung wird der Rezeptor auf der Metalloberfläche immobilisiert und mit dem Target über das angrenzende Dielektrikum in Kontakt gebracht. In einem so genannten Sensorgramm wird die Dimension der Änderung (hier in Pixel) als eine Funktion der Zeit dargestellt (Abb. 1). 


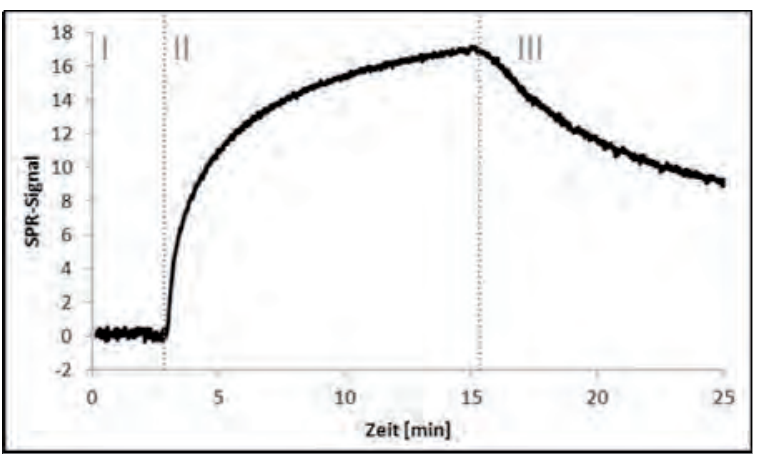

Abbildung 1 Die Phasen eines Sensorgramms. (I) Grundlinie im Pufferfluss. (II) Injektion des Antigens. (III) Ende der Injektion und Spülen mit Puffer.

Im Bereich der klinischen Diagnostik ist der spezifische Nachweis von humanem Serumalbumin (HSA) von entscheidender Bedeutung. HSA ist das von der Leber am stärksten synthetisierte Protein und im Körper u. a. für die Aufrechterhaltung des $\mathrm{pH}$-Wertes sowie für den Transport zahlreicher Substanzen verantwortlich. Darüber hinaus ist das HSA-Level im Plasma ein verlässlicher Marker für die Prognose verschiedener Erkrankungen wie z. B. Lebererkrankungen, Nierenfunktion, Infektionskrankheiten sowie Krebs [2].

Im Rahmen des BMBF-geförderten GO-BIOProjekt „Multi-Organ-Bioreaktoren für die prädiktive Substanztestung im Chipformat" soll HSA zukünftig unter anderem zur Charakterisierung des Zustands von Hepatozyten genutzt werden. Dieser Multi-Organ-Chip, in der Größe eines Objektträgers, soll eine Substanztestung vor einer Exposition am Mensch oder Tier und damit die Vorhersage von relevanten Reaktionen möglich machen [3].

\section{Material und Methoden}

Alle beschriebenen SPR-Experimente wurden mit dem liSPR-System (capitalis technology $\mathrm{GmbH}$ ) bei $30{ }^{\circ} \mathrm{C}$ und einer Fließgeschwindigkeit von $5 \mu \mathrm{L} / \mathrm{s}$ durchgeführt [4], [5].

Die Goldoberfläche der Sensorchips wurde für 30 min mit UV/Ozon (UV/Ozone ProCleaner, NanoAndMore $\mathrm{GmbH}$ ) gereinigt und anschließend mit $10 \mathrm{mmol} / \mathrm{L}$ 11-Mercaptoundecansäure (Sigma-Aldrich Chemie $\mathrm{GmbH}$ ) über Nacht bei $30{ }^{\circ} \mathrm{C}$ funktionalisiert.

Die Immobilisierung des Rezeptors (Anti-HSA Antikörper, Biomol $\mathrm{GmbH}$ ) erfolgte mittels der Carbodiimid-Methode (Amin Coupling Kit, GE Healthcare Europe $\mathrm{GmbH}$ ). Entsprechend den Herstellerangaben wurde die carboxylierte Fläche zunächst für 10 min mit EDC/NHS (1-Ethyl3-(3-(dimethylamino)-propyl)carbodiimide/N-hydroxysuccinimide) aktiviert. Im Anschluss wurden $200 \mu \mathrm{g} / \mathrm{mL}$ Anti-HSA Antikörper für 60 min mit der aktivierten SAM in Kontakt gebracht. Die verbleibenden reaktiven Carboxygruppen wurden im letzten Schritt der Immobilisierung mit Ethanolamin für 30 min abgesättigt. Zur Abschätzung der Spezifität der detektierten Signale ist ein Referenzkanal unabdingbar. Die Referenzfläche sollte dabei so identisch wie möglich zur Messfläche sein, um den Anteil an unspezifischer Bindung sowie Puffereffekte abschätzen zu können. Als Referenz wurde ein Antikörper, der keine Affinität zu HSA oder den Serumbestandteilen besitzt, auf die gleiche Weise wie der Detektionsantikörper immobilisiert.

Nach erfolgter Funktionalisierung und Immobilisierung des Sensorchips wurden HSA-Proben wie beschrieben vermessen: (i) Injektion von $70 \mu \mathrm{L}$ HSA für 10 min. (ii) Spülen mit TBS. (iii) Injektion von $70 \mu \mathrm{L} 2 \mu \mathrm{g} / \mathrm{mL}$ Anti-HSA Antikörper für 10 min. (iv) Spülen mit TBS. (v) Regeneration mit $70 \mu \mathrm{L} 100 \mathrm{mmol} / \mathrm{L}$ Glycine- $\mathrm{HCl}$ pH 2,2 für ca. 2 min.

Als Laufpuffer sowie zur Verdünnung der Proben wurde TBS (Tris Buffered Saline mit Tween $^{\circledR} 20$ pH 8, Sigma-Aldrich Chemie $\mathrm{GmbH}$ ) verwendet.

\section{Ergebnisse und Diskussion}

Zur reproduzierbaren Messung von mehreren Bindungszyklen auf einem Chip muss die Interaktion zwischen Antigen und Antikörper aufgebrochen werden. Die Bindungen werden abhängig von Antikörper-Antigen Reaktion häufig über pH-Änderungen gespalten [1].

In Voruntersuchungen stellte sich $100 \mathrm{mmol} / \mathrm{L}$ Glycine-HCl pH 2,2 als geeignete Regenerationsmethode heraus. Da sich pH-Schocks nachhaltig auf die native tertiäre Struktur der Antikörper auswirken können, muss die Anzahl der möglichen Regenerierungsschritte mittels eines so genannten Scoutings überprüft werden [1]. Dazu wurde $100 \mu \mathrm{g} / \mathrm{mL}$ HSA 10-mal hintereinander injiziert und nachfolgend jeweils mit $70 \mu \mathrm{L} 100 \mathrm{mmol} / \mathrm{L}$ Glycine-HCl pH 2,2 regeneriert. Die nach jeder Injektion gemessenen Bindungssignale sowie die nach jeder Regeneration gemessenen Grundliniensignale wurden gegenübergestellt und ausgewertet.

Die Grundliniensignale bleiben bei allen 10 Zyklen stabil. Dies deutet daraufhin, dass die Regenerierung die bioaktive Sensorschicht nicht nachhaltig beeinträchtigt. Die Bindungssignale hingegen schwanken leicht. So kann erst ab dem zweiten Zyklus von konstanten Signalen ausgegangen werden. Im Folgenden wurden die HSA-Signale aus diesem Grund erst im Anschluss an die erste Regeneration der Oberfläche detektiert. 
Das Detektionslimit des entwickelten Assays liegt bei 2 Pixel (entsprechend einem SignalRausch Verhältnis von 1 zu 3, DIN 32645). Folglich entspricht die HSA-Konzentration von ca. $0,1 \mu \mathrm{g} / \mathrm{mL}$ der Nachweisgrenze gemessen in TBS.

Die minimal detektierbare Konzentration hängt von zahlreichen Faktoren wie unspezifischen Bindungen, Massentransport, der Sensitivität des Sensors sowie von der Antikörper-AntigenBindungseffektivität ab [6].

Um auch geringste Schwankungen des HSALevels der in vitro kultivierten Hepatozyten [3] nachweisen zu können, musste die Sensitivität des entwickelten Assays gesteigert werden. Eine bewährte Methode zur Verstärkung der Sensitivität aber auch der Selektivität ist das Sandwich-Assay [1]. Dabei erfolgt nach Detektion des HSA durch den primären Antikörper ein weiterer Identifizierungsschritt, bei dem ein sekundärer Antikörper hinzugegeben wird. Aufgrund der erhöhten Massenbeladung kommt es entsprechend den Größenverhältnissen von HSA und Antikörper zur Erhöhung des Sensorsignals.

Da es sich bei dem verwendeten Rezeptor um einen polyklonalen Antikörper handelt, kann derselbe Antikörper sowohl als Primär- aber auch als Sekundärantikörper eingesetzt werden.

Mit Hilfe dieser Signalverstärkung konnte die Sensitivität für HSA-Konzentrationen effektiv gesteigert werden.

\section{Zusammenfassung und Ausblick}

Das Ziel des Projektes war es, ein SPRbasiertes Assay zu entwickeln, mit dessen Hilfe HSA-Konzentration in Serumproben spezifisch nachgewiesen werden können. Dazu wurde ein polyklonaler Antikörper mittels CarbodiimidMethode stabil auf den Sensorchip immobilisiert und verschiedene HSA-Konzentrationen vermessen.

Mit einer $\mathrm{pH}-$ Wertänderung konnte die bioaktive Sensorschicht mehrmals regeneriert und so mehrere sequentielle Bindungszyklen reproduzierbar auf einem Chip gemessen werden. Dies gilt als Voraussetzung für die Erstellung von Kalibrierkurven und damit für das Vermessen von Zellkulturüberständen.

Um die Sensitivität des Assays zu steigern wurde ein zweiter Antikörper nach erfolgreicher Bindung des HSA hinzugegeben. Aufgrund der erhöhten Massenbeladung kam es zur gewünschten Signalverstärkung.
In weiterführenden Arbeiten sollen zur weiteren Steigerung der Sensitivität mit Goldnanopartikeln gelabelte Antikörper verwendet werden. Aufgrund der spezifischen Eigenschaften der Goldnanopartikel können die Signale so um mehr als das Achtfache verstärkt werden [7].

Des Weiteren sollen mit Hilfe von Kalibrierkurven Realproben mit unbekannten Antigenkonzentrationen vermessen werden. Über das jeweils detektierte Bindungssignal erfolgt dann die Abschätzung der Konzentrationen. Dazu werden definierte HSAKonzentrationen in denselben Medien gelöst, aus denen später die Realproben bestehen. Nur so kann der Einfluss der verschiedenen Probenbestandteile abgeschätzt und ein aussagekräftiger Zusammenhang zwischen den detektierten SPR-Signalen und den HSAKonzentrationen getroffen werden.

Die spezifische Detektion von Antigenen in Realproben stellt eine besondere Herausforderung dar, da die Antigene zumeist in geringen, die anderen Serumbestandteile in Summe aber in hohen Konzentrationen vorliegen. Um reproduziere Messdaten zu erzeugen, müssen die unspezifischen Bindungen der Serumbestandteile folglich möglichst effizient unterdrückt werden. Zur Erzeugung solcher proteophoben Oberflächen sollen zukünftig Ethylenglykole-basierte Self-assembled Monolayer (SAM) verwendet werden, die für ihre proteinabweisenden Eigenschaften bekannt sind und langzeitstabile bioaktive Schichten auf Goldoberflächen ermöglich. Die Thiolverbindungen ordnen sich dabei aufgrund ihrer Struktur und der Oberfläche so an, dass eine Energieminimierung eintritt. Das ist bei einem einheitlichen Verkippungswinkel gegen die Oberflächennormale gegeben. Um ein optimales Verhältnis an Rezeptordichte und Blockwirkung zu erzeugen, sollen zwei verschieden terminierte Thiolverbindungen verwendet werden. Dabei dient die endständige Funktionalisierung des einen Thiols als Ankerstelle für die Rezeptoren und die des anderen Thiols als Blockung gegen unspezifische Bindungen verwendet werden.

\section{Danksagung}

Die Autoren danken dem Freistaat Sachsen und der Europäischen Union (SAB Projekt UNILOC) für die finanzielle Unterstützung.

\section{Literatur}

[1] D. R. Shankaran, K. V. Gobi, und N. Miura, „Recent advancements in surface plasmon resonance immunosensors for detection of 
small molecules of biomedical, food and environmental interest“, Bd. 121, Nr. 1, S. 158-177, Jan. 2007.

[2] M.-H. Yang, S.-B. Jong, T.-W. Chung, Y.-F. Huang, und Y.-C. Ty, „Quartz crystal microbalance in clinical application“, in Biosensors for Health, Environment and Biosecurity, P. A. Serra, Hrsg. InTech, 2011.

[3] I. Wagner, E.-M. Materne, S. Brincker, U. Süßbier, C. Frädrich, M. Busek, F. Sonntag, D. A. Sakharov, E. V. Trushkin, A. G. Tonevitsky, R. Lauster, und U. Marx, „A dynamic multi-organ-chip for long-term cultivation and substance testing proven by 3D human liver and skin tissue co-culture", Lab. Chip, Bd. 13, Nr. 18, S. 3538-3547, Aug. 2013.

[4] A. Henseleit, S. Schmieder, T. Bley, F. Sonntag, N. Schilling, P. Quenzel, N. Danz, U. Klotzbach, und E. Boschke, „A compact and rapid aptasensor platform based on surface plasmon resonance“, Bd. 11, Nr. 6, S. 573-579, Dez. 2011.

[5] A. Henseleit, S. Schmieder, F. Sonntag, U. Klotzbach, U. Marx, S. Hofmann, und E. Boschke, „SPR-basierte Immunosensoren Universelles Immobilisierungsprotokoll von Antikörpern zur spezifischen AntigenDetektion.", 2011.

[6] J. Xu, D. Suarez, und D. S. Gottfried, „Detection of avian influenza virus using an interferometric biosensor", Anal Bioanal Chem, Bd. 389, Nr. 4, S. 1193-1199, Okt. 2007.

[7] Y. Uludag und I. E. Tothill, „Cancer biomarker detection in serum samples using surface plasmon resonance and quartz crystal microbalance sensors with nanoparticle signal amplification“, Anal. Chem., Bd. 84, Nr. 14, S. 5898-5904, Juli 2012. 